\title{
Stacking and separation of fluorescent derivatives of amino acids by micellar electrokinetic chromatography in the presence of poly(ethylene oxide)
}

\author{
Tai-Chia Chiu ${ }^{a}$, Huan-Tsung Chang a,b,* \\ a Department of Chemistry, National Taiwan University, Taipei, Taiwan \\ ${ }^{\mathrm{b}}$ Department of Natural Science Education, National Taitung University, Taitung, Taiwan \\ Received 25 August 2006; received in revised form 20 January 2007; accepted 26 January 2007 \\ Available online 4 February 2007
}

\begin{abstract}
A new approach for the analysis of large-volume naphthalene-2,3-dicarboxaldehyde (NDA) derivatives of amino acids by micellar electrokinetic chromatography (MEKC) in conjunction with a purple light-emitting diode-induced fluorescence detection is described. In order to optimize resolution, speed, and stacking efficiency, a discontinuous condition is essential for the analysis of NDA-amino acid derivatives. The optimum conditions use 2.0 M TB (pH 10.0) buffer containing $40 \mathrm{mM}$ sodium dodecyl sulfate (SDS) to fill the capillary, deionized water to dilute samples, and $200 \mathrm{mM}$ TB (pH 9.0) containing $10 \mathrm{mM}$ SDS to prepare $0.6 \%$ poly(ethylene oxide) (PEO). Once high voltage is applied, PEO solution enters the capillary via electroosmotic flow and SDS micelles interact and thus sweep the NDA-amino acid derivatives having smaller electrophoretic mobilities than that of SDS micelles in the sample zone. When the aggregates between SDS micelles and NDA amino acid derivatives enter PEO zone, they are stacked due to decrease in electric field and increases in viscosity. Under the optimum conditions, the concentration and separation of $0.53-\mu \mathrm{L} 13$ NDA-amino acid derivatives that are negatively charged has been demonstrated by using a 60-cm capillary, with the efficiencies $0.3-9.0 \times 10^{5}$ theoretical plates and the LODs at signal-to-noise ratio 3 ranging from 0.30 to $2.76 \mathrm{nM}$. When compared to standard injection ( 30 -cm height for $10 \mathrm{~s}$ ), the approach allows the sensitivity enhancements over the range of 50-800 folds for the derivatives. The new approach has been applied to the analysis of a red wine sample, with great linearity of fluorescent intensity against concentrations $\left(R^{2}>0.98\right)$ and the RSD (three repetitive runs in one day) values of the migration times for the ten identified amino acids less than $2.8 \%$.
\end{abstract}

(C) 2007 Elsevier B.V. All rights reserved.

Keywords: Amino acids; Light-emitting diode-induced fluorescence detection; Micellar electrokinetic chromatography; Naphthalene-2,3-dicarboxaldehyde; Polymer solutions; Sodium dodecyl sulfate; Sweeping

\section{Introduction}

Capillary electrophoresis (CE) is a powerful separation technique that possesses a number of advantages, including high separation efficiency, short analysis time, and small amounts of reagents and sample required [1-3]. Although CE using optical detectors has been well demonstrated for the separation of biological molecules such as proteins, nucleic acids, peptides, and amino acids [4-6], its sensitivity is inherent from the limited optical path length (e.g. $75 \mu \mathrm{m})$ and extremely small sample volumes (usually $<10 \mathrm{~nL}$ ) injected to the capillary. Over the past few decades, techniques such as field amplified sample stack-

\footnotetext{
* Corresponding author: Department of Chemistry, National Taiwan University, Taipei, Taiwan. Tel.: +886 2 33661171; fax: +886 233661171.

E-mail address: changht@ ntu.edu.tw (H.-T. Chang).
}

ing, $\mathrm{pH}$-mediate sample stacking, isotachophoresis, sweeping, and use of polymer solutions have been developed for improving the sensitivity [7-13]. Differences in the physicochemical properties (such as $\mathrm{pH}$, ionic strength, and viscosity) of the sample matrix and the background electrolytes are essential for stacking of the analytes. Analyte molecules migrate with faster speed in the sample zone than those in the background electrolyte zone, and thus they are stacked at the boundary either front or back of the sample zone to form sharp sample zones.

Since Quirino and Terabe introduced the concept of sample sweeping almost 10 years ago, sweeping has become one of the most important techniques for improving the sensitivity of analytes, especially neutral solutes $[7,14,15]$. Sweeping involves picking and accumulation of analyte molecules in micellar electrokinetic chromatography (MEKC) in which micelles act as a pseudostationary phase for sweeping the analytes from a long injected sample zone into a narrow band and then for separat- 
ing the concentrated sample zones during electrophoresis [7,14]. Theoretically, neutral analytes having great partition coefficients in micelles can be effectively stacked and separated in MEKC [16,17].

In CE, laser-induced fluorescence (LIF) provides better sensitivity for amino acids than does UV-vis absorption detection. However, besides three aromatic amino acids, most amino acids are not fluorescent in nature. Thus, derivatization of amino acids with reagents to form stable and highly fluorescent derivatives is required in CE-LIF [18-24]. When using a low-cost and purple light-emitting diode (LED) as the light source of $\mathrm{CE}$, naphthalene-2,3-dicarboxaldehyde (NDA) has been found to be practical for derivatization of amino acids. The maximum wavelengths of the two absorption bands of NDA-amino acid derivatives are at 420 and $440 \mathrm{~nm}$ and the emission wavelength is at $490 \mathrm{~nm}$ [25]. Owing to low partition coefficients of NDA-amino acid derivatives in sodium dodecyl sulfate (SDS) and cetyltrimmethylammonium bromide (CTAB), two most commonly used micelles in MEKC, stacking efficiencies and separation resolution in MEKC of the derivatives are not impressive [26]. In order to further improve stacking efficiency and resolution, we tested analysis of amino acids by MEKC in the presence of poly(ethylene oxide) (PEO). When using PEO, SDS is superior over CTAB because SDS micelles migrate into neutral PEO zone, which were expected to achieve great resolution and stacking efficiency based on our previous experiences [27,28].

In this study, we proposed a simple stacking and separation approach for the analysis of NDA-amino acid derivatives by $\mathrm{CE}$ in conjunction with LED induced fluorescence (CELEDIF). Several important electrophoretic parameters such as SDS concentration, PEO concentration, and injection length with respect to separation resolution and stacking efficiency of the CE-LEDIF approach were evaluated. The practicality of the proposed approach was validated by the determination of amino acids in red wine samples.

\section{Experimental}

\subsection{Chemicals}

DL-Amino acids, including alanine (Ala), asparagine (Asn), aspartic acid (Asp), leucine (Leu), cystine (Cys), glutamic acid (Glu), glutamine (Gln), glycine (Gly), histidine (His), serine (Ser), threonine (Thr), tyrosine (Tyr), valine (Val), SDS, and sodium cyanide $(\mathrm{NaCN})$ were obtained from Sigma ( $\mathrm{St}$ Louis, MO, USA). PEO $\left(M_{\mathrm{r}} 8.0 \times 10^{6} \mathrm{~g} \mathrm{~mol}^{-1}\right)$ was obtained from Aldrich (Milwaukee, WI, USA). NDA was obtained from Tokyo Chemical Industry (Tokyo, Japan), and was dissolved in analytical grade methanol that was purchased from Malinckrodt Baker (Phillipsburg, NJ, USA). Sodium tetraborate was obtained from Acros Organic (Geel, Belgium). Tris(hydroxymethyl)aminomethane (Tris) was a product from J.T. Baker (Phillipsburg, NJ, USA). Boric acid was purchased from Riedel-deHaën (Buchs, Switzerland). The stock solution of $400 \mathrm{mM}$ Tris-borate (TB) was prepared by dissolving $24.23 \mathrm{~g}$ of Tris in 500-mL aqueous solution that was adjusted with suit- able amounts of boric acid to $\mathrm{pH} 9.0$. Unless otherwise noted, the molarity of Tris represents that of TB buffer. PEO $(0.015-0.75 \mathrm{~g})$ was gradually added to each of prepared $200 \mathrm{mM}$ TB solutions ( $\mathrm{pH}$ 9.0). During the addition of PEO, a magnetic stirring rod was used to produce a well-homogeneous suspension. After the addition was completed, the solutions were stirred for at least $12 \mathrm{~h}$. Prior to use for CE separation, the solutions were degassed with a vacuum system in an ultrasonic tank for $10 \mathrm{~min}$ [29]. Red wine (Gracia de Chile, Las Condes, Santiago, Chile) was purchased from a local store.

\subsection{Apparatus}

CE-LEDIF system (CE/LIF, Model: 2100) was purchased from Pebio Scientific Company (Taipei, Taiwan). Fused-silica capillary with $75 \mu \mathrm{m}$ I.D. and $365 \mu \mathrm{m}$ O.D. was purchased from Polymicro Technologies (Phoenix, AZ, USA). The capillary length is either $40 \mathrm{~cm}(30 \mathrm{~cm}$ to detector) or $60 \mathrm{~cm}(50 \mathrm{~cm}$ to detector). A DV-E viscometer (Brookfield Engineering Laboratories, Middleboro, MA, USA) was employed to measure the viscosity of PEO solutions in a constant-temperature bath at $25.0 \pm 0.2^{\circ} \mathrm{C}$. All measurements were performed in triplicate.

\subsection{Sample handling and derivatization procedure}

The derivatization procedure of amino acids with NDA in the presence of cyanide was modified from the literature [30]. The derivatization was performed in $1.5-\mathrm{mL}$ centrifuge tubes. For standard NDA-amino acid derivative samples, $1.0-\mathrm{mL}$ reaction mixtures ( $\mathrm{pH} 9.3)$ containing amino acids $(10 \mu \mathrm{M}), \mathrm{NaCN}$ $(0.2 \mathrm{mM})$, NDA $(0.2 \mathrm{mM})$, and sodium tetraborate $(1.0 \mathrm{mM})$ were prepared. For derivatization of amino acids in red wine samples, $1.0-\mathrm{mL}$ reaction mixtures $(\mathrm{pH} 9.3)$ containing $10-\mu \mathrm{L}$ red wine, $\mathrm{NaCN}(0.2 \mathrm{mM})$, NDA $(0.2 \mathrm{mM})$, and sodium tetraborate $(1.0 \mathrm{mM})$ were prepared. The mixtures were allowed to react for $20 \mathrm{~min}$ at room temperature and, if necessary, were diluted to suitable concentrations with deionized water before injecting into the capillary.

\subsection{Procedures}

Prior to analysis, capillaries were filled with high concentration of TB (2.0 M) buffer ( $\mathrm{pH} 10.0)$ containing SDS (0-75 mM). Hydrodynamic injection was applied at $30-\mathrm{cm}$ height (the difference in the exit and entrance ends of the capillary) for 10 to $360 \mathrm{~s}$. After injection, the capillaries were immersed in the PEO solutions and the separations were conducted at $15 \mathrm{kV}$. After each run, PEO in the capillaries were flushed out and the capillaries were equilibrated with $0.5 \mathrm{M} \mathrm{NaOH}$ at $1 \mathrm{kV}$ for $1 \mathrm{~s}$. The capillaries were then filled with TB buffers containing SDS. This treatment was quite successful to regenerate high and reproducible electroosmotic flow $(\mathrm{EOF})(\mathrm{RSD}<1.5 \%)$. During separation, PEO solution that was prepared in TB buffer containing SDS was introduced to the capillary by EOF from the anodic side. The sample volume was estimated according to our previous method by using NDA-amino acid derivatives as a fluorescent marker [31]. The time of the baseline shift indicates 
that the NDA-amino acid derivatives migrated to the detection point, which allows calculation of the sample injection rate $\left(\mathrm{nL} \mathrm{s}^{-1}\right)$. By using the injection rate and injection time, the sample injection volumes were estimated. Stacking enhancement factor (SEF) was used to evaluate quantitatively the stacking efficiency. In this study, SEF is the ratio of the limits of detection (LOD) of the analytes that was injected for $10 \mathrm{~s}$ to that for certain injection times. The LODs of the NDA-amino acid derivatives were estimated at the concentrations that the peak heights generated are three times the noise. The time for the baseline shift due to detection of PEO (neutral) was used to estimate the EOF values [32].

\section{Results and discussion}

\subsection{Schematic representation of stacking and separation of the NDA derivatives of amino acids}

When using PEO solution, EOF gradually decreases as a result of PEO adsorption on the capillary wall [32-34]. In order to reduce $\mathrm{PEO}$ adsorption and thus to generate a high and repeatable EOF, a capillary is filled with $2.0 \mathrm{M}$ TB buffer ( $\mathrm{pH} 10.0$ ) containing 0-75 mM SDS (Fig. 1A). A mixture of NDA-amino acid derivatives is hydrodynamically injected from the anodic end to the capillary for a certain time (e.g. 240 s) (Fig. 1B). Once high voltage is applied, PEO solution enters the capillary from the anodic end. SDS micelles migrating from the zone of $2.0 \mathrm{M}$ TB buffer interact with and thus sweep the negatively charged NDA-amino acid derivatives having smaller electrophoretic

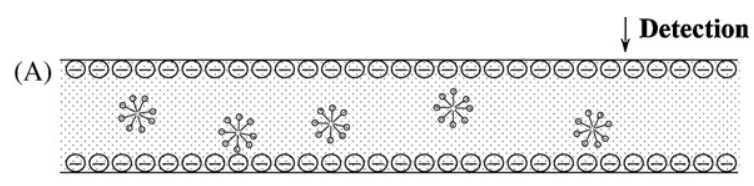

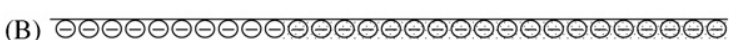
$\square \triangle 0_{0} \Delta \circ \triangle$

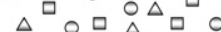

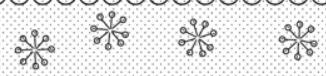

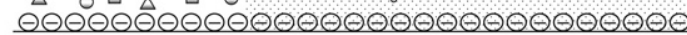
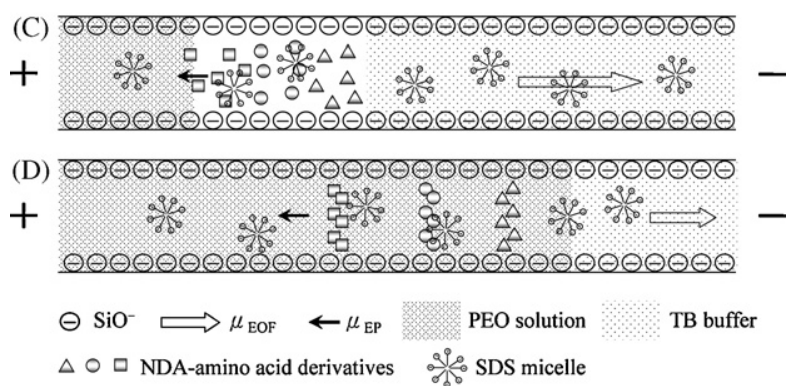

Fig. 1. Evolution of stacking and separation of NDA-amino acid derivatives by CE-LEDIF in the presence of EOF and PEO solutions: (A) The capillary is filled with 2.0 M TB buffer containing SDS, (B) NDA-amino acid derivatives are hydrodynamically injected from anodic end into the capillary for a certain time, (C) when high voltage is applied, SDS micelles sweep the negatively charged NDA-amino acid derivatives in the sample zone. Both SDS micelles and NDAamino acid derivatives migrate against EOF and enter neutral PEO zone during stacking, (D) NDA-amino acid derivatives and the aggregates between SDS micelles and NDA amino acid derivatives are stacked in a narrow band and migrate in the PEO zone. mobilities than that of SDS micelles in the sample zone (Fig. 1C). Both SDS micelles and negatively charged NDA-amino acid derivatives migrate against EOF. For the anionic NDA-amino acid derivatives $\left(1.90-2.65 \times 10^{-4} \mathrm{~cm}^{2} \mathrm{~V}^{-1} \mathrm{~s}^{-1}\right)$ that migrate with lower electrophoretic mobilities than that of SDS micelles $\left(3.43 \times 10^{-4} \mathrm{~cm}^{2} \mathrm{~V}^{-1} \mathrm{~s}^{-1}\right)$ [35], they are picked up by SDS micelles when SDS micelles migrate through the sample zone. When anionic NDA-amino acid derivatives and the aggregates between SDS micelles and NDA amino acid derivatives migrate into the PEO zone, they are stacked as a result of decrease in electric field and increase in viscosity (Fig. 1D). The conductivity values of $2.0 \mathrm{M}$ TB buffer containing $40 \mathrm{mM}$ SDS, sample zone, and PEO solution containing $10 \mathrm{mM}$ SDS are 1500, 3, and $1200 \mu \mathrm{S} \mathrm{cm}^{-1}$. In the presence of SDS, the conductivity values for TB and PEO solutions are even higher. Because PEO solution also contains SDS micelles, the NDA-amino acid derivatives form aggregates with SDS. Finally, the aggregates between SDS micelles and NDA amino acid derivatives are separated according to the mechanisms of MEKC.

\subsection{Effects of SDS in the sample matrix and background electrolyte}

When PEO solutions are used, stacking efficiency, resolution, and speed are dependent on the SDS concentrations, $\mathrm{pH}$ values, and ionic strengths of the sample solutions, background electrolytes (used to fill the capillary), as well as PEO solutions. The impacts of ionic strength and $\mathrm{pH}$ on stacking and separation of small anions have been well studied [36], and they thus are not discussed in this study. In this study, the effects of SDS concentrations can be simply divided to two different categories: (1) control of partition of the NDA-amino acid derivatives between micelles and aqueous phase, and (2) control of EOF through minimization of PEO adsorption. First, we tested the effect of SDS in the sample solution. At $0.6 \%$ PEO that was prepared in $200 \mathrm{mM}$ TB (pH 9.0) containing $10 \mathrm{mM}$ SDS, Fig. 2 exhibits the separations of six NDA-amino acid derivatives $(50 \mathrm{nM})$ that were diluted in deionized water containing different amounts of SDS using a capillary filled with $2.0 \mathrm{M} \mathrm{TB}(\mathrm{pH} 10.0)$ containing $40 \mathrm{mM}$ SDS. The broad peak appearing earlier than those for the analytes in each electropherogram corresponds to the side product of NDA with amino acids. Although NDA is hydrophobic and interacts with the SDS micelles, giving a longer migration time, no signal for NDA-SDS aggregates was observed due to its weak fluorescence. We point out that the injection volume was estimated to be ca. $0.52 \mu \mathrm{L}$ when injecting at $30-\mathrm{cm}$ height for $240 \mathrm{~s}$ using a 40-cm capillary. For the low negatively charged NDA-amino acid derivatives (the first five peaks), the peak efficiencies (1.9-2.2 $\times 10^{5}$ theoretical plates) are not as great as that $\left(9.0 \times 10^{5}\right.$ theoretical plates) for Glu (the last peak) (Fig. 2A). This is mainly because the strongly anionic NDA-Glu derivative migrates with a higher electrophoretic mobility against EOF; it has a lower partition coefficient in SDS micelles than do the low charge-density derivatives and thus its change in mobility is greater when migrating into PEO zone. With increasing SDS concentration in the sample zone, the migrations time for the derivatives become longer, mainly because increases in their 

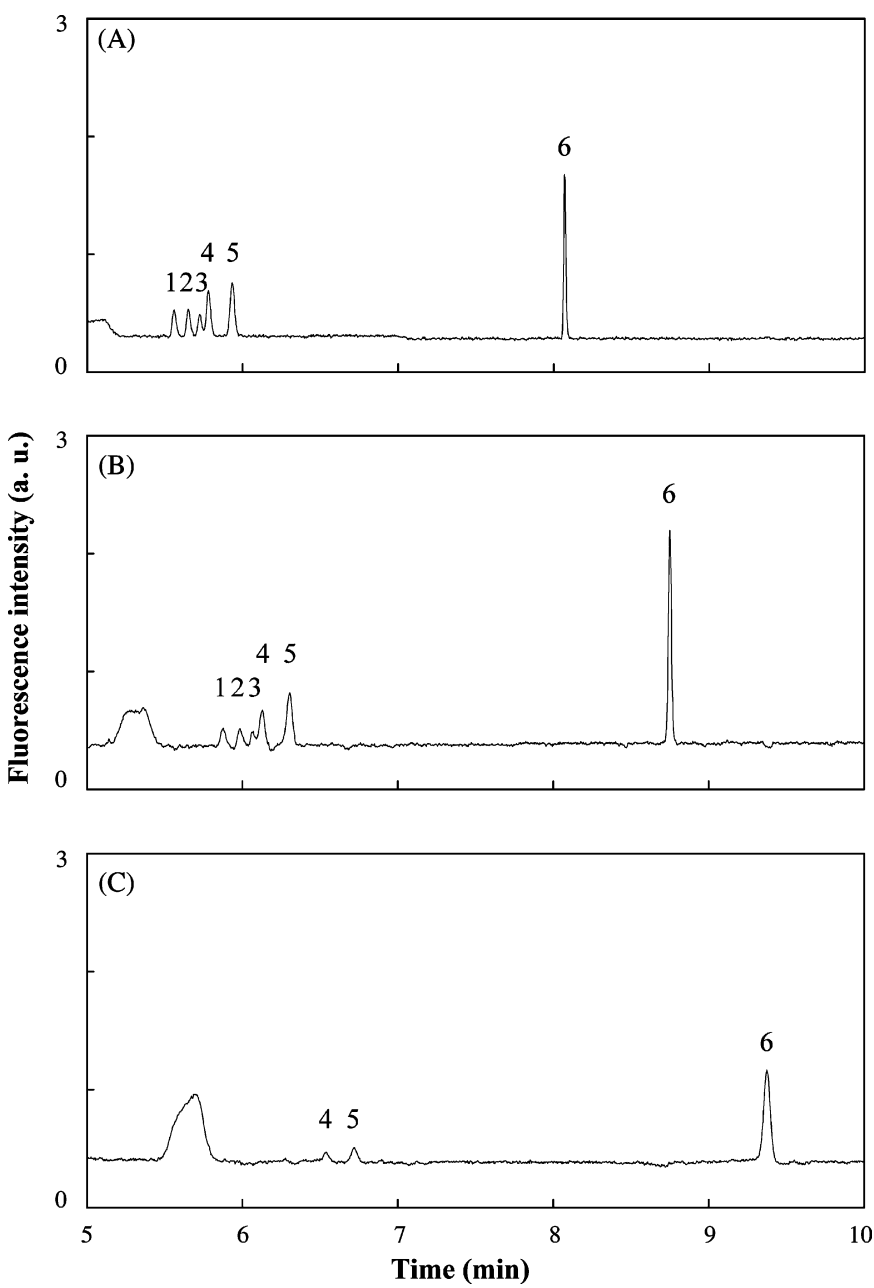

Fig. 2. Impacts of SDS in sample solutions on stacking and separation of NDAamino acid derivatives by CE-LEDIF: (A) No SDS, (B) $0.1 \mathrm{mM}$, (C) $1.0 \mathrm{mM}$, SDS. All sample solutions contained $50 \mathrm{nM}$ NDA-amino acid derivatives. Sample injection was conducted at $30-\mathrm{cm}$ height for $240 \mathrm{~s}$. A 40-cm capillary $(30 \mathrm{~cm}$ of effect length) was filled with $2.0 \mathrm{M} \mathrm{TB}$ (pH 10.0) buffer containing $40 \mathrm{mM}$ SDS prior to sample injection. $0.6 \%$ PEO solution was prepared in $200 \mathrm{mM} \mathrm{TB}$ (pH 9.0) buffer containing $10 \mathrm{mM}$ SDS and separation was conducted at $15 \mathrm{kV}$. Peak identities: NDA derivatives of (1) His, (2) Gln, (3) Asn, (4) Ala, (5) Gly, and (6) Glu.

partition coefficients and increases in the ionic strength of the solution. Owing to increases in the conductivity in the sample zone, the stacking efficiency decreases. As a result, the peaks for the first three derivatives were not detected and the peaks for derivatives $4-6$ become broader and smaller at the SDS concentrations greater than $1.0 \mathrm{mM}$. Based on the result, we suggest that no SDS in the sample matrix is proper.
Next, we conducted the separations of NDA-amino acid derivatives by CE-LEDIF under a similar condition described in Fig. $2 \mathrm{~A}$ besides using a capillary filled with $2.0 \mathrm{M}$ TB buffers (pH 10.0) containing different amounts of SDS. Table 1 summarizes the impacts of SDS concentration on the migration time, peak width, peak height, and theoretical plates of the NDA-amino acid derivatives. The results suggest that the stacking occurring at the boundary between the sample and PEO zones due to increase in viscosity and decrease in electric field is more significant. However, SDS did play a significant role in determining migration time, due mainly to the changes in EOF and partially to the changes in electrophoretic mobility. Using the capillary filled with TB buffers containing $0,0.1$, $1.0,10,20,30,40,50$, and $75 \mathrm{mM}$ SDS, the EOF mobility values were $2.08,2.00,2.33,2.60,3.10,3.58,4.30,4.85$, and $6.17 \times 10^{-4} \mathrm{~cm}^{2} \mathrm{~V}^{-1} \mathrm{~s}^{-1}$, respectively. Because the partition coefficients of the NDA-amino acid derivatives increases with increasing SDS concentration, the changes in migration times for the derivatives are inconsistent with the increases in EOF mobility. We note that SDS micelles migrated against EOF. At the SDS concentrations greater than $40 \mathrm{mM}$, the fluorescence intensities of the derivatives slightly decreased, mainly because of Joule heating $(\sim 50 \mu \mathrm{A})$ and relatively low stacking efficiency. The resolution value between the pair of NDA derivatives of Asn and Ala at 0, 0.1, 1.0, 10, 20,30, 40, 50, and $75 \mathrm{mM}$ SDS were $0.79,0.84,0.66,0.71,0.83,0.92,1.25,1.67$, and 1.60 , respectively. When considering resolution, speed, and stacking efficiency, the optimal buffer for filling the capillary is $2.0 \mathrm{M} \mathrm{TB}$ (pH 10.0) containing $40 \mathrm{mM}$ SDS.

Further, we investigated the effect of SDS concentration in $0.6 \%$ PEO solution on stacking, resolution, and speed under a similar condition. The electropherograms depicted in Fig. 3 display that SDS concentration plays some role in determining stacking, resolution, and speed. In the absence or low SDS concentrations $(<10 \mathrm{mM})$, the peaks are either broad or not well resolved. At high concentrations ( $>12 \mathrm{mM})$, the first three NDAamino acid derivatives co-migrated at the time ( $5.19 \mathrm{~min})$, likely because they all have similar interactions with SDS micelles. At $0,1.0,5.0,8.0,10.0$, and $12.0 \mathrm{mM}$ SDS, the EOF mobilities were 4.04, 4.12, 4.36, 4.36, 4.30, and $4.27 \times 10^{-4} \mathrm{~cm}^{2} \mathrm{~V}^{-1} \mathrm{~s}^{-1}$, respectively. The increase in EOF with increasing SDS concentration is due to decreases in PEO adsorption and the viscosity of PEO solution. The viscosities of $0.6 \%$ PEO solutions containing $0,1.0,5.0,8.0,10.0$, and $12.0 \mathrm{mM}$ SDS are 99.3, 96.9, $90.4,84.2,81.0$, and $74.3 \mathrm{mPa}$ s, respectively. The interactions between PEO and SDS micellar aggregates in aqueous solution cause changes in the size of polymer network and SDS micelles

Table 1

Effect of SDS concentration in 2.0 M TB (pH 10.0) filled into the capillary on peak width, peak height and theoretical plates $(\mathrm{N})$ for stacking and separation of NDA-amino acid derivatives

\begin{tabular}{|c|c|c|c|c|c|c|c|c|c|c|c|c|c|c|c|c|}
\hline & \multicolumn{4}{|c|}{ Migration time (min) } & \multicolumn{4}{|c|}{ Peak width (min) } & \multicolumn{4}{|c|}{ Peak height (a.u.) } & \multicolumn{4}{|c|}{$\mathrm{N}\left(\times 10^{5}\right.$ theoretical plates $)$} \\
\hline & $0^{\mathrm{a}}$ & $10^{\mathrm{a}}$ & $40^{\mathrm{a}}$ & $50^{\mathrm{a}}$ & $0^{\mathrm{a}}$ & $10^{\mathrm{a}}$ & $40^{\mathrm{a}}$ & $50^{\mathrm{a}}$ & $0^{\mathrm{a}}$ & $10^{\mathrm{a}}$ & $40^{\mathrm{a}}$ & $50^{\mathrm{a}}$ & $0^{\mathrm{a}}$ & $10^{\mathrm{a}}$ & $40^{\mathrm{a}}$ & $50^{\mathrm{a}}$ \\
\hline Gly & 12.74 & 9.73 & 5.93 & 4.81 & 0.040 & 0.033 & 0.030 & 0.026 & 1.34 & 1.86 & 1.38 & 1.13 & 5.6 & 4.8 & 2.2 & 1.9 \\
\hline
\end{tabular}

${ }^{\text {a }}$ SDS (mM). 

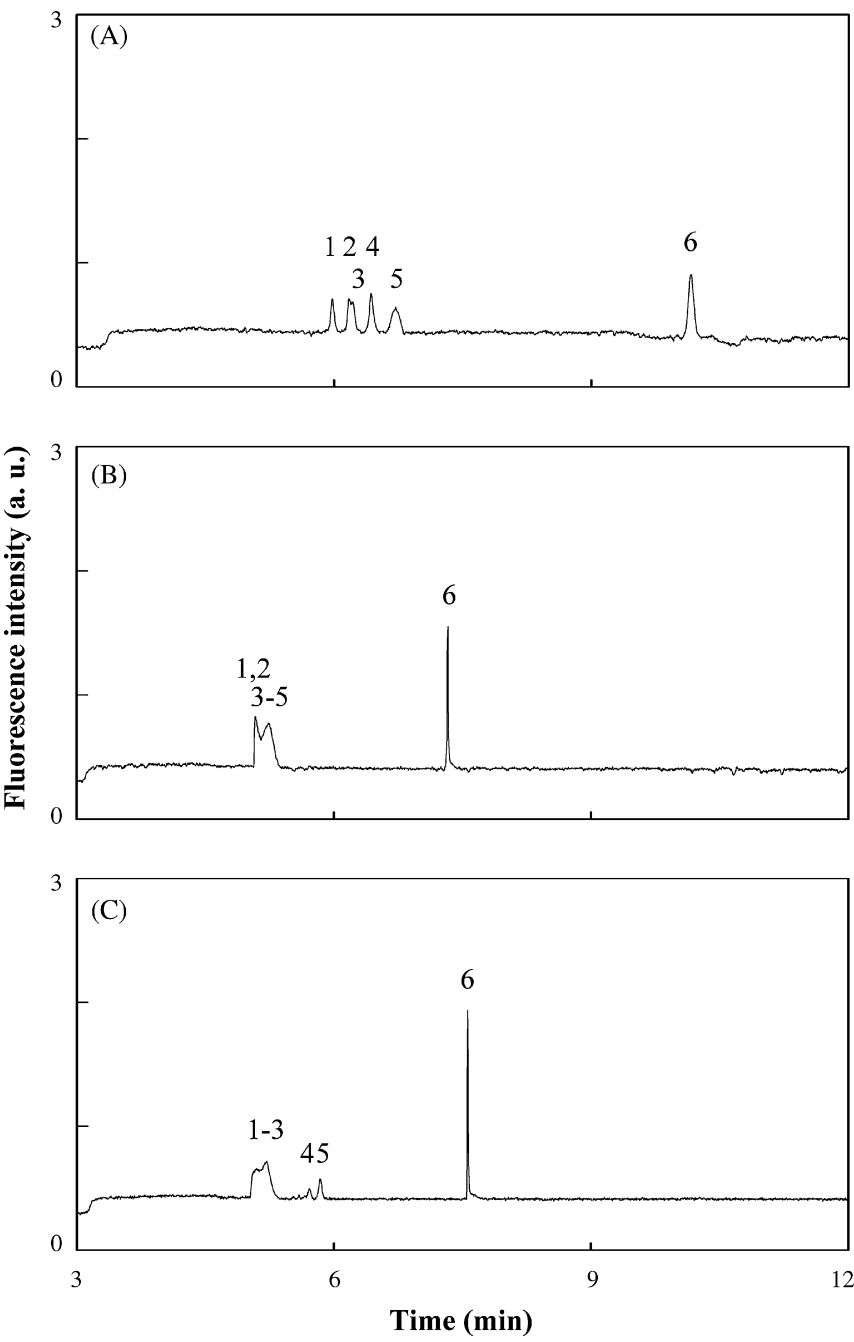

Fig. 3. Electropherograms showing the impact of SDS in $0.6 \%$ PEO solutions on separation and stacking of NDA-amino acid derivatives by CE-LEDIF: (A) No SDS, (B) $8.0 \mathrm{mM}$, and (C) $12 \mathrm{mM}$ SDS, respectively. Other conditions are the same as in Fig. 2A.

as well as the viscosity of the PEO solution [37-39]. At a specific SDS concentration (the critical aggregation concentration $4.1-5.4 \mathrm{mM}$ at $\left.20^{\circ} \mathrm{C}\right)$, SDS aggregates are formed along the polymer chain [37] and their sizes are much smaller than that of the free micelles [39]. The electropherograms depicted in Fig. 3 suggest that $0.6 \%$ PEO prepared in $200 \mathrm{mM} \mathrm{TB}$ (pH 9.0) buffer containing $10 \mathrm{mM}$ SDS is the optimal condition to fill anodic vial for the analysis of NDA-amino acid derivatives.

\subsection{Effect of PEO concentration}

When conducting the separation of NDA-amino acid derivatives by CE-LEDIF in the absence of PEO, it is unsuccessful as depicted in Fig. 4A. The peak profiles of NDA-amino acid derivatives are broad mainly due to poor stacking efficiency and analyte adsorption on the capillary wall. As a result, the separation of the first five NDA-amino acid derivatives was unsuccessful. When compared with the results shown in Figs. 2 and 3, the electropherograms depicted in Fig. 4A-C
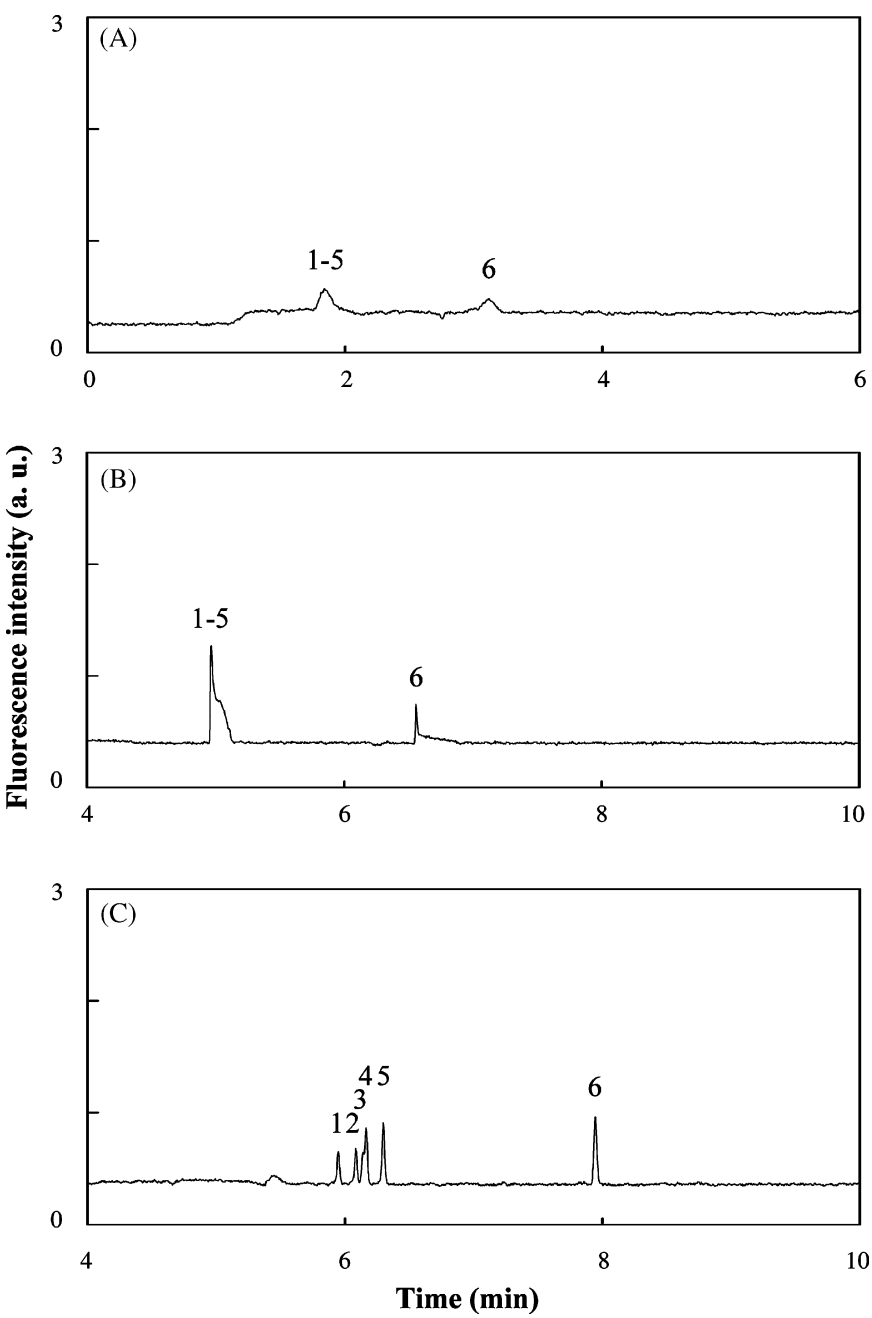

Fig. 4. Effect of PEO concentration on separation and stacking of NDA-amino acid derivatives by CE-LEDIF: (A) $0 \%$, (B) $0.3 \%$, and (C) $1.0 \%$ PEO solutions were prepared in $200 \mathrm{mM}$ TB ( $\mathrm{pH} 9.0$ ) buffer containing $10 \mathrm{mM}$ SDS. Other conditions are the same as in Fig. 2A.

clearly exhibit the role of PEO playing in determining resolution and stacking efficiency. The peaks are unresolved at the PEO concentration less than $0.6 \%$. On the other hand, the resolving power and stacking efficiency decrease with increasing PEO concentration when its concentration is greater than $0.8 \%$, mainly due to strong interactions between PEO and NDA-amino acid derivatives through hydrogen bonding. Since the entanglement threshold concentration of the PEO solution is $0.07 \%$, the PEO solutions all form networks [40,41]. The viscosity values of $0 \%, 0.3 \%, 0.6 \%, 0.8 \%, 1.0 \%$ and $1.5 \%$ PEO solutions containing $10.0 \mathrm{mM}$ SDS are 1.2, 19, 81, 190, 1000, and $7500 \mathrm{mPa}$, respectively. As a result of increases in viscosity and the rate of PEO adsorption with increasing PEO concentration, the EOF mobilities in $0 \%, 0.3 \%, 0.6 \%, 0.8 \%, 1.0 \%$ and $1.5 \%$ PEO solutions containing $10.0 \mathrm{mM}$ SDS were 11.7, 4.94, 4.20, 4.02, 3.65, and $3.01 \times 10^{-4} \mathrm{~cm}^{2} \mathrm{~V}^{-1} \mathrm{~s}^{-1}$, respectively. When comparing the electropherograms depicted in Figs. 3A and 4A, we suggest that PEO concentration is the most important parameter for determining the stacking efficiency. Along with the results shown in Figs. 2-4, we conclude that the optimal stacking and 


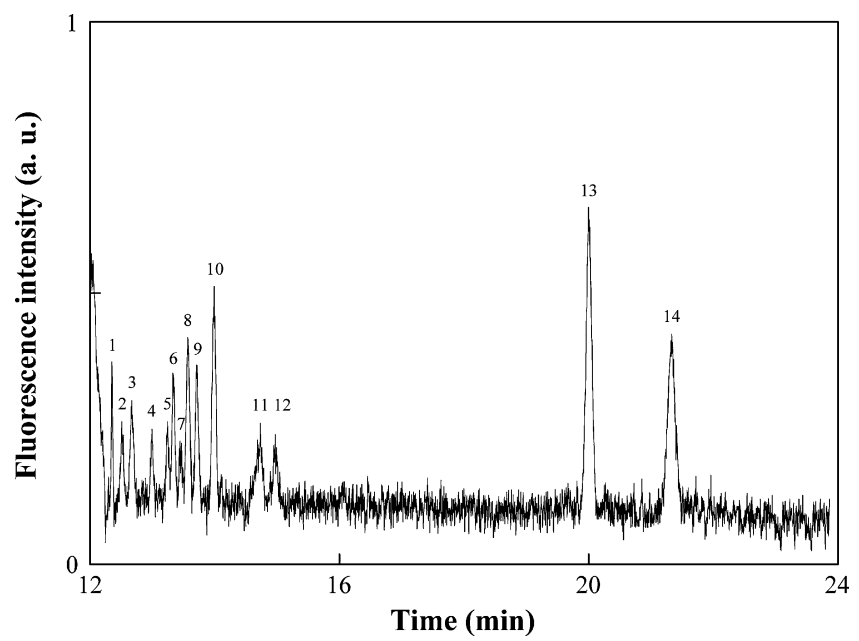

Fig. 5. Analysis of a mixture containing thirteen standard NDA-amino acid derivatives $(5-10 \mathrm{nM})$ by CE-LEDIF using a 60 -cm capillary. Sample solution contained $5 \mathrm{nM}$ NDA-amino acid derivatives, besides $10 \mathrm{nM}$ NDA derivatives of Thr and Cys. Sample injection was performed at 30-cm height for $360 \mathrm{~s}$. Other conditions are the same as in Fig. 2A. Peak identities: NDA derivatives of (1) Leu, (2) Tyr, (3) Vla, (4) His, (5) Gln, (6) Thr, (7) Asn, (8) Ser, (9) Ala, (10) Gly, (11) D-Cys, (12) L-Cys, (13) Glu, and (14) Asp.

separation conditions are using 2.0 M TB (pH 10.0) buffer containing $40 \mathrm{mM}$ SDS to fill the capillary, deionic water to dilute samples, and $0.6 \%$ PEO that was prepared in $200 \mathrm{mM} \mathrm{TB}(\mathrm{pH}$ 9.0) containing $10 \mathrm{mM}$ SDS to fill the anodic vial.

\subsection{Separation of 13 standard NDA-amino acid derivatives}

Fig. 5 exhibits the analysis of a mixture containing thirteen standard NDA-amino acid derivatives (5-10 nM) by CE-LEDIF using a 60-cm capillary under the optimal conditions. The injection volume was estimated to be ca. $0.53 \mu \mathrm{L}$ when injecting at $30-\mathrm{cm}$ height for $360 \mathrm{~s}$. All 13 derivatives were resolved within $22 \mathrm{~min}$ although the injection volume is $0.53 \mu \mathrm{L}$. Based on the electropherogram, we estimated the LODs $(\mathrm{S} / \mathrm{N}=3)$ of Leu, Tyr, Val, His, Gln, Thr, Asn, Ala, Ser, Gly, D-Cys, L-Cys, Glu, and Asp were 1.18, 1.52, 0.81, 1.22, 0.96, 1.03, 1.18, 0.46, 0.50, 0.38, $2.76,2.50,0.30$, and $0.71 \mathrm{nM}$, respectively. The efficiencies and sensitivity enhancements for the derivatives are in the ranges of $0.3-9.0 \times 10^{5}$ theoretical plates and 50 to 800 folds, respectively, showing effective stacking. To test the quantitative practicality of this approach, different concentrations $(5-100 \mathrm{nM})$ of the NDA-amino acid derivatives were subjected to CE-LIF analyses. The linearity $\left(R^{2}>0.98\right)$ for each NDA-amino acid derivative is good, showing the potential of this approach for the analysis of amino acids.

\subsection{Analysis of red wine}

To test the practicality of the proposed approach, the determination of amino acids in red wine samples was performed. Fig. 6 exhibits a representative electropherogram of separating a $0.18-\mu \mathrm{L}$ diluted red wine sample under the optimal separation conditions. The RSD values ( 3 repetitive runs) of the migration times for the identified amino acids are $<2.8 \%$. The day-to-day

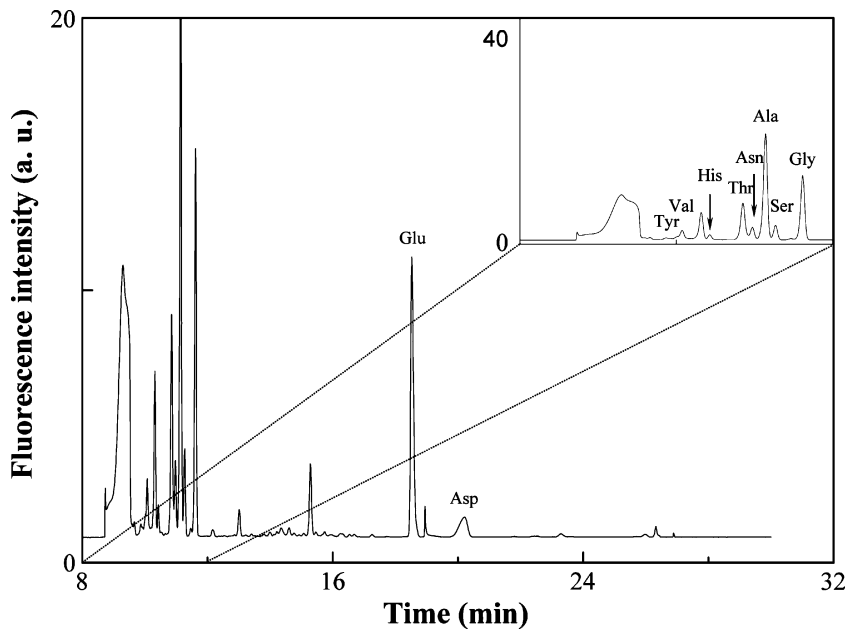

Fig. 6. Electropherogram of separating a $0.18-\mu \mathrm{L}$ diluted red wine sample. The analysis conditions are the same as in Fig. 5.

(five consecutive days) and capillary-to capillary (three different capillaries) RSD values were less than $3.0 \%$ and $3.5 \%$, respectively. Since NDA only form fluorescent derivatives with solutes possessing primary amino groups, all the unidentified peaks are mainly corresponding to amines and amino acids having primary amino groups. Because CE-LEDIF was applied, fewer peaks were detected than those were by applying $\mathrm{CE}$ with UV-vis absorption detection. In addition, the LIF detection is also at least 3-order magnitudes more sensitive than is UV-vis absorption. We also point out that small peaks such as those for Tyr and His were not detected without applying the stacking technique. By performing an internal standard method (peak height), we estimated the concentrations of Tyr, Val, His, Thr, Asn, Ala, Ser, Gly, Glu, and Asp in the red wine sample to be $3.11 \pm 0.23,4.01 \pm 0.38,1.44 \pm 0.09,28.5 \pm 1.35$, $4.47 \pm 0.33,29.2 \pm 1.84,5.81 \pm 0.42,18.7 \pm 1.28,36.8 \pm 2.68$, and $6.87 \pm 0.56 \times 10^{-5} \mathrm{M}$ ( 3 repetitive runs). The results suggest that this method has a great potential for the analysis of amino acids, especially trace ones, in beverages.

\section{Conclusions}

We reported a stacking and separation method in MEKC in the presence of PEO solutions for the analyses of NDA-amino acid derivatives and a red wine sample. We have found that, in addition to SDS, PEO is essential for the stacking and separation of large-volumes (e.g. $0.53 \mu \mathrm{L}$ ) NDA-amino acid derivatives. When compared to neutral analytes, anionic NDA-amino acid derivatives under the separation conditions have lower partition coefficients between SDS micelles and aqueous solution. As a result, the stacking and separation of 13 NDA-amino acid derivatives by MEKC in the absence of PEO was unsuccessful. Under optimized conditions, the thirteen NDA-amino acid derivatives were well separated by MEKC in the presence of $0.6 \% \mathrm{PEO}$ after injection of $0.53 \mu \mathrm{L}$. The LODs for thirteen amino acids range from 0.30 to $2.76 \mathrm{nM}$, with the sensitivity enhancements over the range of 50-800 folds. The successful analysis of a red wine sample further suggest that the practicality of the present 
approach. Based on our results, we conclude that the present approach can be applied to analysis of large-volume samples containing neutral and anionic solutes.

\section{Acknowledgements}

This work was supported by the National Science Council of Taiwan under contract numbers NSC 95-2113-M-002-026MY3. T.-C.C. is grateful to the National Science Council for his postdoctoral fellowship in the Department of Chemistry, National Taiwan University, under contract number NSC 952811-M-002-051.

\section{References}

[1] Y.-W. Lin, T.-C. Chiu, H.-T. Chang, J. Chromatogr. B 793 (2003) 37.

[2] C.W. Huck, R. Bakry, G.K. Bonn, Electrophoresis 27 (2006) 111.

[3] J. Kraly, M.A. Fazal, R.M. Schoenherr, R. Bonn, M.M. Harwood, E. Turner, M. Jones, N.J. Dovichi, Anal. Chem. 78 (2006) 4097.

[4] H.-T. Chang, Y.-F. Huang, S.-H. Chiou, T.-C. Chiu, M.-M. Hsieh, Curr. Protomics 1 (2004) 325.

[5] W.W.C. Quigley, N.J. Dovichi, Anal. Chem. 76 (2004) 4645.

[6] P.R. Powell, A.G. Ewing, Anal. Bioanal. Chem. 382 (2005) 581.

[7] J.P. Quirino, S. Terabe, Science 282 (1998) 465.

[8] D.M. Osbourn, D.J. Weiss, C.E. Lunte, Electrophoresis 21 (2000) 2768.

[9] S. Sentellas, L. Puignou, M.T. Galceran, J. Sep. Sci. 25 (2002) 975.

[10] S.D. Arnett, C.E. Lunte, Electrophoresis 24 (2003) 1745.

[11] M. Urbanek, L. Krivankova, P. Bocek, Electrophoresis 24 (2003) 466.

[12] M.-M. Hsieh, T.-C. Chiu, W.-L. Tseng, H.-T. Chang, Curr. Anal. Chem. 2 (2006) 17.

[13] Y. Yang, R.I. Boysen, M.T.W. Hearn, Anal. Chem. 78 (2006) 4752.

[14] J.P. Quirino, S. Terabe, Anal. Chem. 71 (1999) 1638.

[15] J.P. Quirino, S. Terabe, K. Otsuka, J.B. Vincent, G. Vigh, J. Chromatogr. A 838 (1999) 3.

[16] J.P. Quirino, J.-B. Kim, S. Terabe, J. Chromatogr. A 965 (2002) 357.
[17] C.-W. Huang, H.-P. Jen, R.-D. Wang, Y.-Z. Hsieh, J. Chromatogr. A 1110 (2006) 240

[18] R.M. Latorre, S. Hernandez-Cassou, J. Saurina, J. Chromatogr. A 934 (2001) 105 .

[19] S. Hillebrand, J.R. Schoffen, M. Mandaji, C. Termignoni, H.P.H Grieneisen, T.B.L. Kist, Electrophoresis 23 (2002) 2445.

[20] M. Ummadi, B.C. Weimer, J. Chromatogr. A 964 (2002) 243.

[21] H. Li, H. Wang, J.-h. Chen, L.-h. Wang, H.-s. Zhang, Y. Fan, J. Chromatogr B 788 (2003) 93.

[22] S. Oguri, M. Hibino, M. Mizunuma, Electrophoresis 25 (2004) 1810.

[23] M.-J. Lu, T.-C. Chiu, P.-L. Chang, H.-T. Ho, H.-T. Chang, Anal. Chim. Acta 538 (2005) 143.

[24] M.T. Veledo, M. de Frutos, J.C. Diez-Masa, J. Chromatogr. A 1079 (2005) 335.

[25] P. de Montigny, J.F. Stobaugh, R.S. Givens, R.G. Carlson, K. Srinivasachar, L.A. Sternson, T. Higuchi, Anal. Chem. 59 (1987) 1096

[26] H.-J. Shen, C.-H. Lin, Electrophoresis 27 (2006) 1255.

[27] W.-L. Tseng, Y.-W. Lin, H.-T. Chang, Anal. Chem. 74 (2002) 4828.

[28] Y.-F. Huang, M.-M. Hsieh, W.-L. Tseng, H.-T. Chang, J. Proteome Res. 5 (2006) 429

[29] S.-H. Chiou, M.-F. Huang, H.-T. Chang, Electrophoresis 25 (2004) 2186

[30] P.-L. Chang, T.-C. Chiu, H.-T. Chang, Electrophoresis 27 (2006) 1922.

[31] W.-L. Tseng, M.-M. Hsieh, S.-J. Wang, C.-C. Huang, Y.-C. Lin, P.-L. Chang, H.-T. Chang, J. Chromatogr. A 927 (2001) 179.

[32] W.-L. Tseng, H.-T. Chang, Anal. Chem. 72 (2000) 4805.

[33] W.-L. Tseng, M.-M. Hsieh, S.-J. Wang, H.-T. Chang, J. Chromatogr. A 894 (2000) 219.

[34] T.-C. Chiu, H.-T. Chang, J. Chromatogr. A 979 (2002) 299.

[35] M.R. Karim, S. Shinagawa, T. Takagi, Electrophoresis 15 (1994) 1141.

[36] M.-M. Hsieh, C.-E. Hsu, W.-L. Tseng, H.-T. Chang, Electrophoresis 23 (2002) 1633.

[37] L.M. Smitter, J.F. Guedez, A.J. Muller, A.E. Saez, J. Colloid Interf. Sci 236 (2001) 343.

[38] K. Chari, J. Kowalczyk, J. Lal, J. Phys. Chem. B 108 (2004) 2857.

[39] R. Meszaros, I. Varga, T. Gilanyi, J. Phys. Chem. B 109 (2005) 13538.

[40] S.P. Radko, A. Chrambach, J. Phys. Chem. 100 (1996) 19461.

[41] C.-C. Huang, Y.-F. Huang, H.-T. Chang, J. Nanosci. Nanotechnol. 4 (2004) 622. 